\title{
Interception de la pluie par un mulch de feuilles de canne à sucre (Saccharum officinarum): utilisation d'un dispositif de mesure automatisé pour le test d'un modèle
}

\author{
F Bussière 1, P Cellier 2 \\ 1 INRA, station agropédoclimatique de la zone Caraibe, Unité de bioclimatologie, \\ BP 1232, F97185 Pointe-à-Pitre Cedex, Guadeloupe; \\ 2 INRA, station de bioclimatologie, 78850 Thiverval-Grignon, France
}

(Received 15 March 1992; accepted 12 October 1992)

\begin{abstract}
Résumé - La fraction des pluies interceptées par un mulch végétal puis perdues par évaporation peut être importante en cas de pluies faibles. Pour estimer précisément ces pertes en fonction du régime pluviométrique et des caractéristiques du mulch, il est nécessaire de savoir paramétrer les différents processus impliqués. Pour cela, un dispositif automatique permettant la mesure simultanée des différents termes du bilan hydrique d'un mulch de feuilles de canne à sucre a été réalisé. Il a été utilisé pour évaluer un modèle semi-empirique d'interception de la pluie établi pour un couvert forestier. La mesure directe du stock d'eau contenu dans le mulch a permis pour la première fois de tester différentes hypothèses de ce modèle. Le mulch, d'un indice foliaire de 4,0, présente une capacité de stockage de $2,8 \mathrm{~mm}$. La majorité des pluies enregistrées pendant l'expérimentation n'a pas saturé le mulch, ce qui a rendu difficile la vérification de sa cinétique d'égouttage. Les relations simples de calcul de l'évaporation du couvert mouillé semblent validées par nos données. Cependant, un nombre plus important de pluies serait nécessaire pour confirmer ces résultats. La modélisation validée par ce système devrait pouvoir être étendue à des couverts végétaux.
\end{abstract}

mulch / canne à sucre / interception de la pluie / mesure automatique / bilan hydrique

Summary - Rainfall interception by a mulch of sugar cane leaves: use of an automatic measurement device to test a model. Water losses due to rainfall interception by vegetation can be important, especially in the case of a crop residue mulch because of its high water storage capacity. The proportion of interception losses is related both to mulch characteristics (LAl, structure, water retention by leaves) and to climatic conditions (rainfall quantity, intensity and duration). In order to accurately estimate these losses, it is necessary to describe all the involved processes as correctly as possible. An automatic device for measuring several components of the water balance of a mulch of sugarcane leaves was developed. Direct measurements of mulch water storage and drainage enabled us to check different assumptions made in most rainfall interception models. The mulch with a leaf area index of 4.0 had a water storage capacity of $2.8 \mathrm{~mm}$. Most of the rain events measured during the experiment did not saturate the mulch. Therefore checking the simulation of its dripping kinetics has been difficult. Simulation of evaporation of the saturated canopy is more accurate. However, this test should be completed by using a rainfall simulator in order to obtain a large range of rainfall intensities and durations. After further validation, the rainfall interception model could be extended to the case of vegetation canopies.

mulch / sugarcane / rainfall interception / automatic measurement / water balance 


\section{INTRODUCTION}

En milieu tropical, dans les zones à saison sèche marquée, la forte demande évaporative et les ressources en eau limitées ont amené les agriculteurs à développer des techniques culturales spécifiques visant la réduction de ces contraintes (Stigter, 1987). L'une de ces techniques consiste à laisser sur le sol, voire à rapporter, des résidus de récoltes qui forment un mulch naturel. Outre la limitation du ruissellement, les principaux effets recherchés sont la limitation des pertes hydriques par évaporation et la réduction des températures du sol. A la Guadeloupe (Antilles françaises), les feuilles à canne à sucre, laissées sur les parcelles après la récolte, forment naturellement un mulch. La culture n'étant pas couvrante pendant les 5 mois suivants, celui-ci peut jouer un rôle significatif dans le bilan hydrique du sol.

Le mulch forme un écran isolant à la surface du sol et va donc modifier les transferts radiatifs, la diffusion de la chaleur et de la vapeur d'eau entre le sol et l'atmosphère et intercepter une partie des précipitations. Mais cet impact n'est pas facile à estimer, car si le mulch limite l'évaporation, les précipitations qu'il peut capter sont directement évaporées dans l'atmosphère sans réalimenter le sol sous-jacent.

Cet effet a parfois été évoqué dans diverses études empiriques (Brun et al, 1986; Midmore et al, 1986) mais jusqu'à présent, l'influence réelle du mulch sur le bilan hydrique n'a pas été quantifiée. II est nécessaire pour cela de connaître précisément les processus d'interception de la pluie afin de pouvoir les paramétrer en fonction de variables météorologiques et de caractéristiques du mulch.

Après un rappel des approches du phénomène d'interception de la pluie trouvées dans la bibliographie, nous présentons un dispositif expérimental original qui nous a permis de tester diverses hypothèses et paramétrisations classiquement utilisées dans les modèles semiempiriques.

\section{L'INTERCEPTION DE LA PLUIE : MÉCANISMES ET PRINCIPALES COMPOSANTES}

La plupart des études portent sur des couverts forestiers (on pourra consulter les revues bibliographiques de Zinke (1967) et Blake (1975)) car ceux-ci, avec leur grande surface végétale développée, peuvent intercepter une proportion importante des précipitations ( 25 à $70 \%$ d'après Leyton et al, 1967). Le bilan hydrique concernant l'eau libre présente à la surface du couvert peut s'écrire :

$$
\mathrm{d} C / \mathrm{d} t=P-D-E
$$

avec $P$ : pluie incidente (précipitations arrivant sur le couvert); $D$ : pluie nette ou drainage (précipitation totale atteignant le sol après traversée du couvert); $C$ : stock d'eau contenu dans le couvert; $E$ : évaporation; $t$ : temps.

Dans le cas d'un couvert forestier, la pluie nette peu se décomposer de la manière suivante :

$$
D=\mathrm{p} P+E_{g}+E_{t}
$$

avec $p$ : proportion de pluie incidente atteignant directement le sol sans être interceptée; $E_{g}$ : pluie atteignant le sol après avoir été momentanément interceptée par le couvert; $E_{t}$ : pluie interceptée par le couvert atteignant le sol après écoulement le long des branches puis du tronc.

$\mathrm{Ce}$ dernier terme pouvant représenter un volume d'eau important pour certains arbres (Aussenac, 1970), devient négligeable dans le cas d'un couvert de blé (Butler et Huband, 1985) et inexistant pour des résidus végétaux placés à la surface du sol.

La pluie nette $D$ et le stock d'eau $C$ peuvent être influencés par l'intensité de la pluie ellemême, mais cet effet est assez controversé. Ainsi, Schulze et al (1978) montrent que la quantité maximale d'eau interceptée par un couvert forestier croît avec l'intensité de la pluie incidente. En revanche, des expérimentations effectuées en laboratoire par Mohamoud et Ewing (1990) sur des résidus de maïs et soja montrent que ce paramètre a peu d'effet sur $C$.

Un autre terme fréquemment introduit est la capacité maximale de stockage du couvert (S), atteinte une fois la pluie achevée $(P=D=0)$. Aussenac et Boulangeat (1980) ont montré expérimentalement que $S$ dépendait de l'espèce et, d'après Aston (1979), au sein de celle-ci, elle est proportionnelle à l'indice foliaire. Merriam (1961), sur un couvert de Raygrass, met en évidence le rôle de la hauteur de la culture. L'exemple de l'eucalyptus pour lequel $S$ est faible en raison de la position verticale des feuilles, témoigne de l'importance de l'inclinaison des surfaces foliaires 
(Crockford et Richardson, 1990a). Les propriétés physiques des surfaces réceptrices elles-mêmes (mouillabilité) sont déterminantes : Barfield et al (1973) ont montré que, pour différentes plantes maraîchères, ce paramètre peut varier du simple au double.

De manière générale, peu de travaux concernent l'interception de la pluie par des cultures basses (McMillan et Burgy, 1960; Couturier et Ripley, 1973; Butler et Huband, 1985) ou des végétaux morts (Pitman, 1989; Mohamoud et Ewing, 1990). Dans ces conditions, il n'est pas possible d'obtenir une estimation de la quantité d'eau interceptée par le mulch à partir de données bibliographiques:

- celui-ci, planophile et ayant une forte densité de surface foliaire est trop différent des couverts les plus étudiés;

- le volume d'eau intercepté dépendant simultanément de paramètres liés au couvert et des conditions météorologiques est très variable (de 5 à $80 \%$ d'après Horton, 1919).

\section{MODÈLES D'INTERCEPTION DE LA PLUIE}

\section{Modèles empiriques}

Ce sont des ajustements statistiques entre le volume de la pluie incidente $(P)$ et la pluie nette $(D)$ ou la variation de stock d'eau de la canopée (C), à l'échelle de l'averse.

Les ajustements effectués peuvent être :

- des régressions linéaires, de la forme $D=a P$ + b ou $C=a P+b$ (Zinke, 1967; Gash, 1979; Crockford et Richardson, 1990b);

- des ajustements polynomiaux ou logarithmiques (Bultot et al, 1972; Aussenac, 1968), fonction hyperbolique (Calheiros de Miranda et Butler, 1986) ou exponentielle (Couturier et Ripley, 1973; Pitman, 1989; Pook et al, 1991).

Compte tenu de la variabilité du phénomène que nous venons d'évoquer, ce type de modèle fonctionne pour un couvert en un lieu donné et des conditions météorologiques particulières (celles de l'expérience).

\section{Modèles semi-empiriques}

Ces modèles prennent en compte des cinétiques élémentaires de variation de stock d'eau du couvert au cours du temps. Dans une série d'articles, Rutter et al $(1971,1975,1977)$ proposent un modèle d'interception permettant d'évaluer tous les termes du bilan hydrique $(C$, $D$ et $E$, d'après les définitions données en introduction) pour un pas de temps de 5 min (Rutter et al, 1971). Puis des simplifications sont proposées, permettant l'utilisation de ce modèle pour une large gamme de couverts forestiers, sur des pas de temps plus importants, compatibles avec des mesures des variables d'entrée plus sommaires (Rutter et al, 1975). Des tests de ce modèle ont par la suite été effectués par différents auteurs (Gash et Morton, 1978; Gash et al, 1979). Dans un souci de simplification et pour une mise en œuvre opérationnelle, Gash (1979) propose une modélisation où la pluie est représentée par une seule averse journalière, sur laquelle les différents termes du bilan sont appliqués successivement. Enfin, Jiagang (1988) propose un modèle théorique utilisant un formalisme inspiré de la modélisation des transferts radiatifs qui calcule l'interception par tranche de canopée.

Jackson (1975) ayant testé, pour une forêt en climat tropical, différents modèles semiempiriques, conclut que seuls ceux basés sur une description fine des phénomènes comme celui de Rutter et al (1971) conservent un caractère prédictif.

\section{MATÉRIEL ET MÉTHODE}

\section{Le modèle de Rutter et al (1971)}

Il effectue un bilan hydrique du couvert en calculant, à chaque pas de temps, les différents termes de gain et de perte d'eau. Ce bilan peut être résumé par l'équation suivante :

$$
-\mathrm{d} C / \mathrm{d} t=\mathrm{K} \exp \{\mathrm{b} C\}-(1-p) P+E
$$

avec $C$ : quantité d'eau stockée dans le couvert $(\mathrm{mm}) ; P$ : pluie incidente $\left(\mathrm{mm} \cdot \mathrm{h}^{-1}\right) ; E$ : évaporation $\left(\mathrm{mm} \cdot \mathrm{h}^{-1}\right) ; K, b$ : coefficients de drainage du couvert; $\rho$ : coefficient de traversée du couvert (proportion de la pluie traversant directement, sans toucher la végétation).

Le premier terme à droite de l'équation (3) est l'égouttage de l'eau interceptée par le couvert auquel Rutter et al (1971) ont donné une forme exponentielle. Le deuxième représente la fraction de la pluie incidente interceptée par celui-ci.

L'évaporation de l'eau contenue dans le mulch, E, est calculée de la façon suivante : 
- Si $C \geq S, E=E_{p}$

- Si $C<S, E=E_{p} C / S$

avec $E_{p}$ : évaporation potentielle (mm.h-1).

Le fonctionnement du modèle nécessite donc la connaissance, au pas de temps requis, 1) de variables météorologiques permettant de calculer les variables de forçage : $E_{p}$ et $P, 2$ ) de paramètres dépendants du couvert : $K, b, p, S$.

La plupart des paramétrisations, même si elles ont été souvent employées, n'ont pas toujours été très bien établies. II est souvent difficile de mesurer expérimentalement certaines variables en milieu forestier $(C$ et $S$ par exemple). Certaines hypothèses, comme la cinétique d'égouttage et la paramétrisation de l'évaporation employées dans le modèle, n'ont jamais été vérifiées; c'est ce que nous nous proposons de faire dans la suite de cette étude, dans le cas d'un mulch végétal.

\section{Dispositif expérimental}

L'expérimentation a été réalisée au centre INRA de Guadeloupe, au domaine Duclos $\left(16^{\circ} 15^{\prime} \mathrm{N}, 61^{\circ} 40^{\prime} \mathrm{W}\right.$, altitude $125 \mathrm{~m}$ ). Sur une parcelle de $10 \times 20 \mathrm{~m}$, on a rapporté un mulch composé de feuilles de canne à sucre dont les dimensions caractéristiques sont $1 \mathrm{x}$ $0,05 \mathrm{~m}$ environ. Celui-ci présente un indice foliaire de 4,0 sur une dizaine de centimètres d'épaisseur. Au sein de cette parcelle, un dispositif permet la mesure simultanée de la pluie incidente, de la pluie percolant à travers le mulch et de la quantité d'eau stockée dans celui-ci (fig 1). Une cage en aluminium de $5 \mathrm{~m}^{2}$ pour $0,1 \mathrm{~m}$ d'épaisseur, contenant un tapis de mulch, est régulièrement pesée à l'aide d'un système fonctionnant de la manière suivante : la cage est reliée par un câble pouvant coulisser librement à un contrepoids de masse supérieure placé sur une balance pouvant être déplacée suivant un axe vertical à l'aide d'une plateforme hydraulique. Ces mouvements sont pilotés par un ordinateur (Husky Hunter 208) qui assure si-

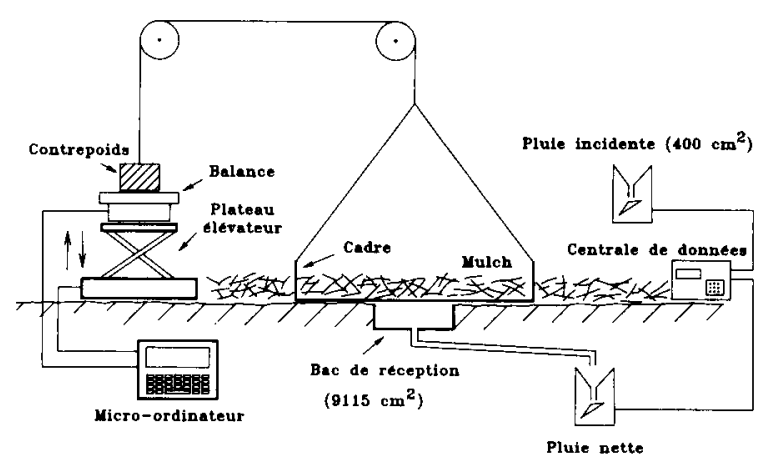

Fig 1. Représentation schématique du dispositif expérimental permettant simultanément la mesure des pluies incidentes et nettes et du stock d'eau du mulch. multanément l'enregistrement des pesées. Les pluies incidentes et percolant à travers le cadre de mulch sont mesurées grâce à des pluviomètres à augets standards (Précis mécanique) munies de surfaces réceptrices respectivement de 400 et $9115 \mathrm{~cm}^{2}$.

Nous disposons donc des mesures suivantes:

- pluie incidente et percolante mesurées toutes les minutes avec une précision de $0,2 \mathrm{~mm}$ et de $0,014 \mathrm{~mm}$, respectivement;

- stock d'eau du mulch mesuré toutes les demi-heures afin de ne pas trop perturber les conditions naturelles par des soulèvements trop fréquents (précision de $0,02 \mathrm{~mm}$ ).

Les données météorologiques ont été mesurées avec un pas de temps trihoraire à une centaine de mètres du dispositif expérimental par une station agrométéorologique automatique Cimel (Paris, France). Celles-ci permettent de calculer $E_{p}$ à partir de la formule de Penman (1948) pour laquelle le rayonnement atmosphérique est calculé à partir de la formule de Brunt (1952). Les valeurs semi-horaires de $E_{p}$ sont estimées à partir des données météorologiques trihoraires, en supposant une évolution sinusoïdale de celles-ci au cours de la journée.

\section{RÉSULTATS}

\section{Performances globales du dispositif}

Les mesures présentées dans cette étude ont été effectuées de novembre 1989 à avril 1990, ce qui correspond à une fin de saison des pluies et un début de saison sèche à la Guadeloupe. On présente sur la figure 2 les enregistrements effectués du 21 au 24 novembre, qui illustrent les performances du système et l'ensemble des situations pouvant être décrites par celui-ci. Le mulch est initialement très sec quand surviennent les pluies dans la nuit du 21 au 22. Une forte pluie (plus de $5 \mathrm{~mm}$ ) va porter le stock d'eau du mulch à $3,6 \mathrm{~mm}$, mais celui-ci retombe très vite à $2 \mathrm{~mm}$ dans l'après-midi, du fait de l'évaporation, malgré de petites pluies inférieures au millimètre. II est intéressant de remarquer que les petites averses du 23 aux alentours de minuit ramènent le stock d'eau du mulch à une valeur restant stable jusqu'au lever du jour. Cette valeur de $3 \mathrm{~mm}$ mesurée en absence d'évaporation est proche de la capacité maximale de rétention du mulch. Le lendemain, le stock redescend à une valeur de $0,5 \mathrm{~mm}$, qui augmentera jusqu'à $1 \mathrm{~mm}$ au lever du jour, du simple falt de la condensation nocturne. Cette dernière observation montre la finesse des observations possible avec ce dispositif. 


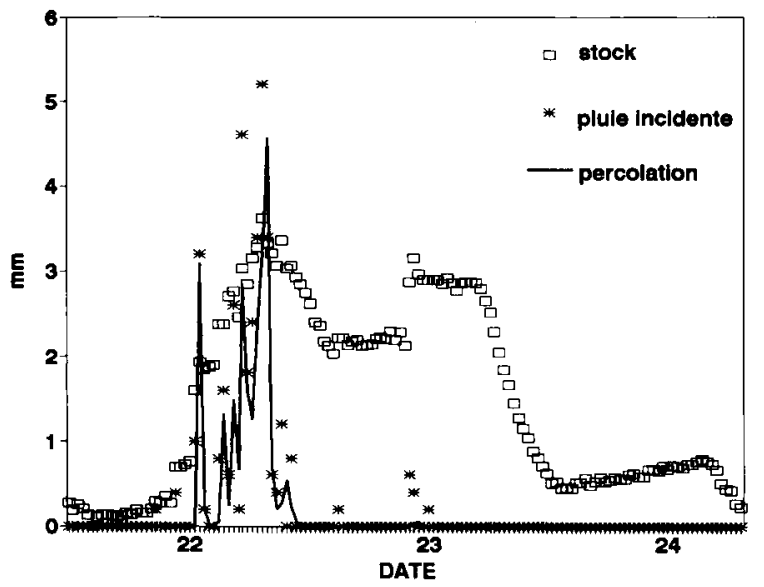

Fig 2. Exemple d'enregistrement effectué par le dispositif, du $21 / 11 / 1989$ à $12 \mathrm{~h}$ au 24/11/1989 à $12 \mathrm{~h}$ : variation de stock d'eau du mulch, de la pluie incidente et de la pluie nette au pas de temps de la demi-heure.

\section{Relation globale entre la pluie nette et la pluie incidente}

La figure 3 représente l'ensemble des données que nous avons obtenues, soit 109 pluies dont $80 \%$ sont inférieures à $3 \mathrm{~mm}$, valeur proche de la capacité de stockage du couvert. Dans ce type de relation (Leyton et al, 1967; Rutter et al, 1971; Jackson, 1975; Pearce et Rowe, 1981), on ne conserve habituellement que les pluies survenant sur un mulch sec, afin de s'affranchir de la variabilité due aux conditions initiales. Mais, disposant d'une estimation indépendante de ce stock, nous avons pu porter toutes les pluies sur cette figure, en ajoutant à la pluie incidente le stock d'eau initial. On obtient alors une relation tout à fait comparable à celles publiées, et présentant une dispersion plus faible. En effet, la variabilité observée par ces auteurs est causée par la différence des conditions d'évaporation qui prévalent pendant et après la pluie (tant qu'il y a percolation). Dans le cas de pluies faibles, les durées de la pluie et de l'égouttage sont réduites et les conditions d'évaporation jouent un rôle moindre dans le bilan d'interception. Nous avons cependant distingué, comme cela est classique dans ce type d'étude (Jackson, 1975), les pluies nocturnes (ayant lieu en l'absence d'évaporation) et les pluies diurnes, pour lesquelles la variabilité des conditions d'évaporation entraîne une variation de la quantité totale d'eau interceptée par le couvert durant un épisode pluvieux.

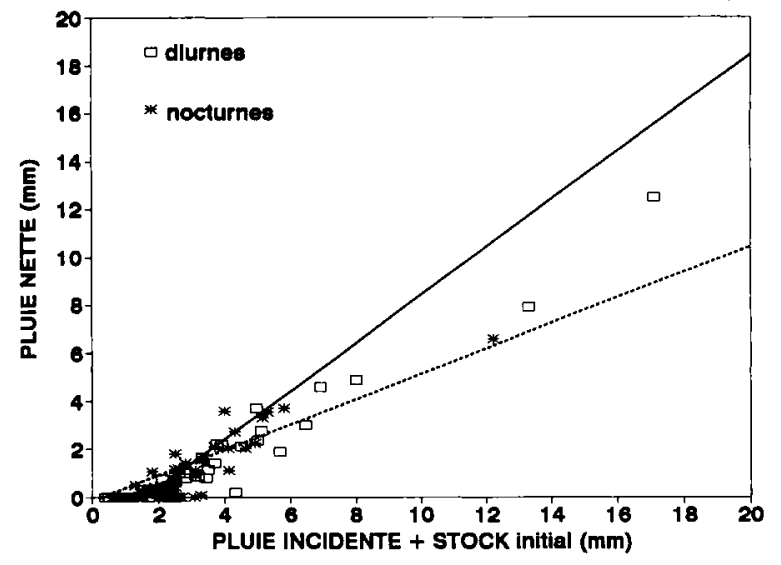

Fig 3. Relation entre la pluie nette et la pluie interceptée pour l'ensemble des données obtenues de novembre 1989 à avril 1990. La droite en trait continu représente les valeurs maximales de pluies nettes obtenues pour des pluies incidentes nocturnes dépassant la capacité de stockage du mulch ( $>3,5 \mathrm{~mm}$ ), en pointilié, l'ajustement aux moindres carrés pour les pluies nocturnes insuffisantes pour saturer le mulch $(<2,5 \mathrm{~mm})$.

\section{Calcul de S, capacité de stockage du couvert}

Ce paramètre a été calculé par la méthode classique employée par les utilisateurs du modèle de Rutter et al (1971) rappelée par Gash et Morton (1978). Nous avons sélectionné pour cela les pluies survenues en condition d'évaporation négligeable (nocturnes) et susceptibles de dépasser la saturation du mulch (supérieures à 3,5 $\mathrm{mm}$ ). Parmi ces pluies, celles représentant la pluie nette maximale pour une même pluie incidente caractérisent les situations où l'évaporation a été la plus faible pendant la pluie et donc le stock d'eau du mulch maximal. La droite passant par ces points et de pente unitaire est généralement appelée "enveloppe" et son abscisse à l'origine représente la capacité de stockage du couvert. Cette droite a pour équation :

$$
D=P-1,6 \text { soit } S \geq 1,6 \mathrm{~mm}
$$

Cependant, compte tenu du nombre restreint de données et du caractère subjectif de la notion d'«enveloppe", cette mesure est sujette à caution. Nous pouvons obtenir une estimation beaucoup plus fiable de $S$ à partir des pesées du stock d'eau contenu dans le mulch effectuées toutes les demi-heures. Sur l'ensemble des mesures, nous avons repéré les pluies nocturnes suffisantes pour dépasser la capacité de stockage du mulch estimée, en première approxima- 
tion, à $3 \mathrm{~mm}$, et nous avons noté le stock restant lorsque la pluie ayant cessé, la percolation s'arrête à son tour. Nous obtenons ainsi 7 valeurs, qui donnent :

$$
S=2,8 \pm 0,5 \mathrm{~mm} \text {. }
$$

Cette valeur est plus élevée que celles obtenues pour des couverts forestiers $(0,8$ et 1,1 pour Gash et Morton, 1978 et Rutter et al, 1971 respectivement) même dans le cas de forêts tropicales : 0,89 pour Jackson (1975) et 1 pour Pearce et Rowe (1981).

Elle est cependant tout à fait comparable au 2,13 trouvé par Leyton et al (1967) pour un tapis de fougères d'un indice foliaire de 3,56 ayant de plus un port planophile comme le mulch.

Nous constatons qu'en raison de la faiblesse de l'échantillonnage et du type de pluies (averses successives de faible intensité), la méthode proposée par Gash et Morton (1978) donne une mauvaise estimation de la capacité de stockage du mulch.

Nous avons également comparé cette valeur à celle qui résulte de la mesure de la capacité de rétention des feuilles de mulch, comme cela a été présenté par Nizinski et Saugier (1988) : pesée des feuilles que l'on vient de tremper dans l'eau puis passage à l'étuve $\left(80^{\circ} \mathrm{C}\right)$. On obtient ainsi la capacité de rétention maximale par surface unitaire de feuilles : $0,13 \mathrm{~mm} / \mathrm{m}^{2} \pm 0,018$ (17 échantillons).

Cette valeur est supérieure à celle obtenue par Crockford et Richardson (1990b) pour des feuilles d'eucalyptus probablement plus lisses et recouvertes de cire : $0,04 \mathrm{~mm} / \mathrm{m}^{2}$. En revanche, Nizinski et Saugier (1988) ont obtenu une capacité de rétention plus importante pour des feuilles de chêne : $0,47 \mathrm{~mm} / \mathrm{m}^{2} \pm 0,23$ (29 échantillons).

Le mulch ayant un indice foliaire total de 4,0 , nous obtenons une capacité de rétention de : $0,13 \times 4,0=0,52 \mathrm{~mm}$. Cette valeur, très inférieure à la valeur réelle, est liée à la structure particulière du matériau : les couverts végétaux sont formés de feuilles disjointes réparties dans l'espace, tandis que les éléments de feuillage du mulch ont de nombreux points de contact entre eux. Cette disposition va permettre la formation de ménisques entre les éléments de feuillage proches. De plus, près de la surface, il peut y avoir un éclaboussement de certaines faces inférieures de feuilles lors de l'interception par une feuille voisine, ce qui augmente d'autant la capa- cité de stockage du couvert. D'autre part, les feuilles principalement planophiles et la structure relativement rigide de l'ensemble limitent l'égouttage spontané. Ces propriétés particulières vont toutes dans le sens d'une augmentation de la capacité maximale de rétention du mulch.

\section{Calcul de la proportion de pluie traversant directement le couvert, $\mathrm{p}$}

Le mulch étudié représentant un couvert dense et couvrant totalement le sol (indice foliaire de 4,0 réparti sur $0,1 \mathrm{~m}$ d'épaisseur), on peut supposer que $p=0$. Gash et Morton (1978) proposent, pour estimer $p$, d'utiliser les pluies nocturnes insuffisantes pour saturer le couvert : dans ces circonstances, la pluie nette est composée uniquement des précipitations traversant directement le couvert sans rencontrer de feuille. La pente de la droite de régression entre la pluie incidente et la pluie nette nous donne donc la valeur du paramètre $p$ (pointillés fig 3 ). Cette méthode appliquée à nos valeurs expérimentales donne:

$$
D=0,53( \pm 0,04) P-0,17( \pm 0,2) \quad\left(r^{2}=0,75\right)
$$

(en pointillés fig 3 ), soit $p=0,53$, résultat, là encore, en contradiction évidente avec le taux de couverture du sol.

Notre dispositif nous ayant permis l'enregistrement simultané des pluies incidentes et nettes, nous pouvons suivre l'évolution du cumul, à l'échelle de chaque averse, de la pluie nette en fonction de la pluie incidente cumulée. L'ensemble des données nocturnes est représenté sur la figure 4, l'échelle logarithmique en abscisse permettant de mieux discerner les évolutions en début de pluie. Systématiquement au début de chaque pluie, on retrouve une période pendant laquelle l'eau ne s'écoule pas encore à travers le mulch, alors qu'une pluie incidente est enregistrée. Cela confirme l'hypothèse selon laquelle le mulch est couvrant et ne laisse pas passer directement de précipitations. La zone sans pluie nette au début de chaque averse varie entre 0,4 et $2,2 \mathrm{~mm}$ suivant le stock d'eau initial contenu dans le mulch.

\section{Calcul des paramètres $K$ et $b$}

Lorsqu'il ne pleut pas, mais que le mulch continue de s'égoutter, en l'absence d'évaporation 


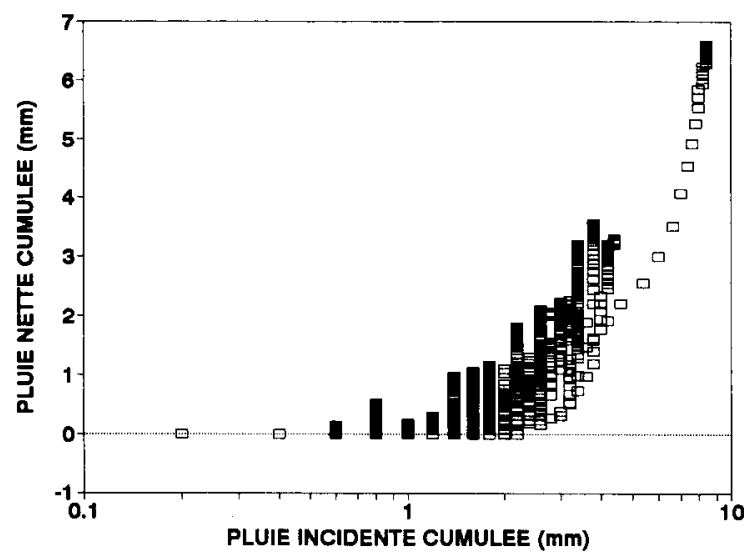

Fig 4. Évolution de la pluie nette en fonction de la pluie incidente pour l'ensemble des averses nocturnes (pas de temps: $1 \mathrm{~min}$ ). Une échelle logarithmique est utilisée en abscisse.

(conditions nocturnes), l'équation de bilan du modèle de Rutter et al (1971) devient :

$$
-\mathrm{d} C / \mathrm{d} t=\mathrm{K} \exp \{\mathrm{b} C\}
$$

Les pesées nous permettent d'avoir une estimation du stock d'eau au début de la pluie ( $C$ initial). Pour l'ensemble des précipitations remplissant les conditions précédentes et compte tenu de la résolution de nos enregistrements $(0,013$ $\mathrm{mm} / \mathrm{min}$ ), nous avons considéré que la cinétique de percolation était correctement restituée pour des volumes allant jusqu'à $0,039 \mathrm{~mm} / \mathrm{min}$. En deçà, le caractère discret de la mesure risque d'introduire un biais trop important, l'écoulement de l'auget n'étant comptabilisé qu'à la fin du remplissage de celui-ci. Pour chacune de ces périodes de drainage, les paramètres $K$ et $b$ peuvent alors être calculés par un algorithme d'ajustement non linéaire (méthode de Marquart) (SAS, 1987).

Dix-sept cinétiques d'égouttage nous ont permis de calculer les paramètres suivants : $K=$ $0,004 \pm 0,005$ et $b=2,3 \pm 0,9$.

L'ensemble des ajustements est présenté sur la figure 5; on obtient une famille de fonctions exponentielles avec une forte variabilité du coefficient $\mathrm{K}$. II semble que les fortes valeurs de $\mathrm{K}$ correspondent à un $\mathrm{C}$ initial faible. Ce modèle, vérifié par Rutter et al (1971), pour des conditions de pluies saturant le couvert $(P>>S)$ semble ne pas être adapté aux pluies faibles.

Pour un couvert forestier, Rutter et al (1971) ont trouvé la valeur $b=3,7$ ce qui est logique : pour un même stock d'eau, le couvert végétal,

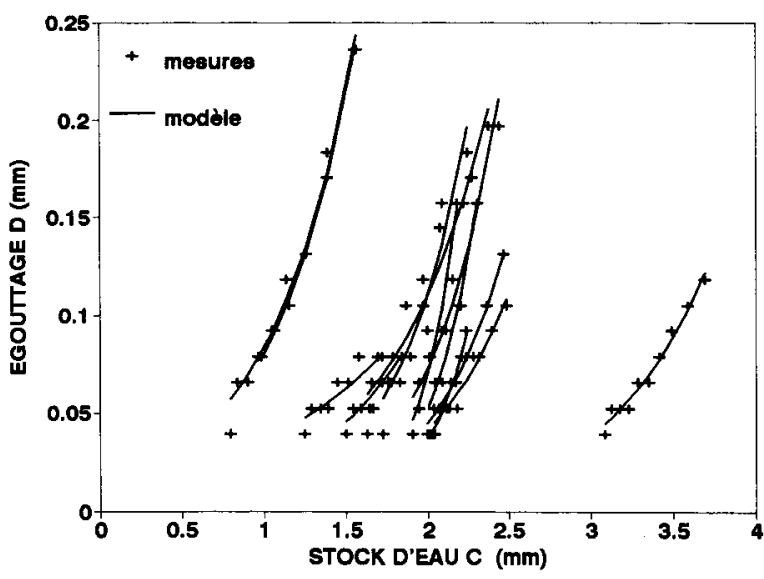

Fig 5. Relation entre la pluie nette persistant en l'absence de pluie incidente (égouttage du mutch) et le stock d'eau du mulch (C) pour l'ensemble des averses nocturnes. Données expérimentales et modèle de Rutter et al (1971) ajusté.

moins compact, draine plus facilement que le mulch.

On note de plus les tendances suivantes : $\mathrm{K}$ et $b$ sont systématiquement liés par un coefficient de corrélation inférieur à $-0,99$. En outre, la relation donnée par Rutter et al (1971) $(K=r$ $\exp \{-\mathrm{bS}\}$ où $r=$ résolution de la mesure $=0,039$ $\mathrm{mm}$ ) sous-estime systématiquement $\mathrm{K}$.

\section{Vérification de la relation $\mathrm{E}=\mathrm{E}_{\mathrm{p}} \mathrm{C} / \mathrm{S}$}

En général, l'absence de mesure de stock d'eau du couvert ne permet pas la vérification de cette relation. L'ensemble des données est présenté sur la figure 6 . La droite représente la relation $E=E_{p} C / S$ (avec $S=2,8 \mathrm{~mm}$ ). Nous avons éga-

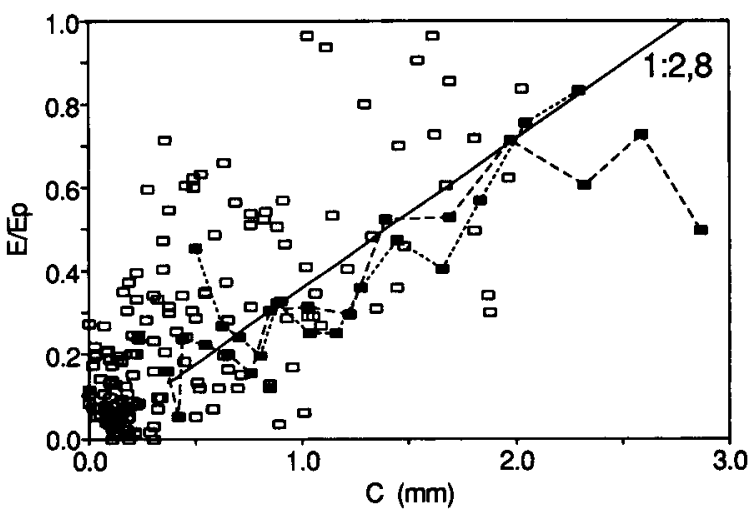

Fig 6. Relation entre le rapport $E / E_{p}$ (E étant mesurée par pesées et Ep calculée à partir de donnés météorologiques) et le stock d'eau du mulch (C), la droite représente $E / E_{p}=\mathrm{C} /$ $\mathrm{S}$. Les symboles pleins représentent l'évolution après deux fortes pluies (ayant saturé le mulch). 
lement figuré l'évolution du rapport $E / E_{p}$ pour deux pluies importantes (supérieures à $S$ ) suivies d'une période de dessèchement bien marquée sur une journée. L'évolution de l'évaporation est alors proche de celle simulée par le modèle théorique, ce qui tend à valider celui-ci. Pour l'ensemble des points restants, une grande partie de la variabilité est due à de petites pluies qui réhumectent le mulch, sans pour autant atteindre la capacité de stockage $S$, et augmentent fortement l'évaporation pendant des durées courtes.

\section{CONCLUSION}

Notre dispositif expérimental a permis l'acquisition de données précises à des pas de temps courts des différents termes du bilan hydrique d'un mulch liés à l'interception de la pluie. Cet outil peut permettre la mesure du phénomène d'interception de la pluie sur des mulchs de différentes natures ou des couverts de dimensions réduites. II nous a permis de tester diverses hypothèses du modèle de Rutter et al (1971) concernant linterception, la cinétique d'égouttage et l'évaporation du couvert et d'estimer directement les paramètres $S$ et $p$. La forme exponentielle de la relation liant le drainage à la quantité d'eau présente dans le mulch n'avait à notre connaissance pas été testée directement. Cette relation semble effectivement suivre une variation exponentielle mais les coefficients $K$ et $b$ censés ne dépendre que de caractéristiques propres au couvert pourraient être sensibles aux conditions initiales et à l'intensité de la pluie. Seule une expérimentation mettant en jeu différents régimes de pluies permettrait de conclure sur ce point.

La dernière hypothèse testée concerne le calcul d'évaporation du couvert, émise par Rutter et al (1971), qui a été reprise telle quelle par la plupart des auteurs (Gash, 1979; Hancock et Crowther, 1979). Cette hypothèse d'évaporation proportionnelle au stock d'eau contenu dans le mulch semble être vérifiée.

Compte tenu des aléas climatiques, il semble indispensable de coupler ce système de mesure à un simulateur de pluies, il serait alors possible d'explorer rapidement une large gamme de pluviométries et de propriétés physiques du mulch (indice foliaire, épaisseur, capacité de rétention) indispensable pour initier et valider une modélisation fine du phénomène.

Les perfectionnements du dispositif expérimental envisagés devraient permettre la validation de cinétiques de percolations appropriées. Nous étudions un matériau pour lequel les différents termes du bilan hydrique sont facilement mesurables. Une modélisation basée sur l'analyse des phénomènes élémentaire d'interception au niveau des éléments de feuillage peut être validée dans le cas d'un mulch et transposable pour une culture.

\section{RÉFÉRENCES}

Aston AR (1979) Rainfall interception by eight small trees. J Hydrol 42, 383-396

Aussenac $\mathrm{G}$ (1968) Interception des précipitations par le couvert forestier. Ann Sci Forest 25 (3), 135-156

Aussenac G (1970) Action du couvert forestier sur la distribution au sol des précipitations. Ann Sci Forest 27 (4), 383-399

Aussenac G, Boulangeat C (1980) Interception des précipitations et évapotranspiration réelle dans des peuplements de feuillu (Fagus silvatica $\mathrm{L}$ ) et de résineux (Pseudostuga menziesii (Mirb) Franco). Ann Sci Forest 37 (2), 91-107

Barfield BJ, Payne FA, Walker JN (1973) Surface water storage capacity of selected crop leaves under irrigation sprays. Agric Meteorol 12, 105-111

Blake GJ (1975) The interception process. In: National Symposium on Hydrology (Austral Acad Sci, ALT Chapman, Dunin, eds) Canberra 59-81

Brun LJ, Enz JW, Larsen JK, Fanning C (1986) Springtime evaporation from bare and stubblecovered soil. J Soil Water Cons 41 (2) 120-122

Brunt D (1952) Radiation in the troposphere. Physical and dynamical meteorology. University Press, Cambridge, 124-142

Bultot F, Dupriez GL, Bodeux A (1972) Interception de la pluie par la végétation forestière : estimation de l'interception journalière à l'aide d'un modèle mathématique. J Hydrol 17, 193-223

Butler DR, Huband NDS (1985) Throughfall and stemflow in wheat. Agric For Meteorol 35, 329-338

Calheiros de Miranda RA, Butler DR (1986) Interception of rainfall in a hedgerow apple orchard. $J H y$ drol 87, 245-253

Couturier DE, Ripley EA (1973) Rainfall interception in mixed grass prairie. Can J Plant Sci 53, 659-663

Crockford RH, Richardson DP (1990a) Partitioning of rainfall in a eucalypt forest and pine plantation in Southeastern Australia. II. Stemflow and factors affecting stemflow in a dry sclerophyll eucalypt forest and a Pinus radiata plantation. Hydrol Proc 4, 145-155

Crockford RH, Richardson DP (1990b) Partitioning of rainfall in a eucalypt forest and pine plantation in Southeastern Australia. IV. The relationship of interception of these forests, and the effect on interception of thinning the pine plantation. Hydrol Proc 4, 168-188 
Gash JHC (1979) An analytical model of rainfall interception by forests. Quart J $A$ Meteorol Soc 105, 43-55

Gash JHC, Morton AJ (1978) An application of the Rutter model to the estimation of the interception loss from Thetford forest. J Hydrol 38 (1-2), 49-58

Gash JHC, Lloyd CR, Stewart JB (1979) SIM5T/12- a model of forest transpiration and interception using data from an automatic weather station. In: Comparison of forest water and energy exchange models (S Halldin, ed). International Society for Ecological Modelling, Copenhagen, 173-184

Hancock NH, Crowther JM (1979) A technique for the direct measurement of water storage on a forest canopy. J Hydrol 41, 105-122

Horton RE (1919) Rainfall interception. Mon Weather Rev 47(9), 603-623

Jackson IJ (1975) Relationships between rainfall parameters and interception by tropical forest. $J$ Hydrol $24,215-238$

Jiagang $L$ (1988) A theoretical model of the process of rainfall interception in forest canopy. Ecol Model 42 (2), 111-123

Leyton L, Reynolds ERC. Thompson FB (1967) Rainfall interception in forest and moorland. Proceedings of the international symposium on forest hydrology (Sepper EW, Lull HSW, eds). Pergamon Press, Oxford, 163-178

McMillan WD, Burgy RH (1960) Interception loss from grass. J Geophys Res 65 (8), 2389-2394

Merriam RA (1961) Surface water storage on Annual Ryegrass. J Geophys Res 66 (6), 1833-1838

Midmore DJ, Berrios D, Roca J (1986) Potato (Solanum spp) in hot tropics II soil temperature and moisture modification by mulch in contrasting environments. Field Crops Res 15, 97-108

Mohamoud YM, Ewing LK (1990) Rainfall interception by corn and soybean residue. Trans ASAE 33 (2), 507-511

Nizinski J, Saugier B (1988) Mesures et modélisation de l'interception nette dans une futaie de chênes. Acta $C_{\text {Ecol }}$ Ecol Plant 9(3), 311-329
Pearce AJ, Rowe LK (1981) Rainfall interception in a multi-storied, evergreen forest: estimates using Gash's analytical model. J Hydrol 49, 341-353

Penman HL (1948) Natural evaporation from open water, bare soil and grass. Proc Roy Soc A, Lond $193,120-145$

Pitman Jl (1989) Rainfall interception by bracken litter-relationship between biomass, storage and drainage rate. J Hydrol 111 (1/4), 281-291

Pook EW, Moore PHR, Hall T (1991) Rainfall interception by trees of Pinus radiata and Eucalyptus viminalis in a $1300 \mathrm{~mm}$ rainfall area of southeastern New South Wales. I. Gross losses and their variability. Hydrol Proc 5, 127-141

Rutter AJ, Morton AJ (1977) A predictive model of rainfall interception in forests. III. Sensitivity of the model to stand parameters and meteorological variables. J Appl Ecol 14, 567-588

Rutter AJ, Morton AJ, Robins PC (1975) A predictive model of rainfall interception in forests. II. Generalization of the model and comparison with observations in some coniferous and hardwood stands. $J$ Appl Ecol 12, 367-380

Rutter AJ, Kershaw KA, Robins PC, Morton AJ (1971) A predictive model of rainfall interception in forests. I. Derivation of the model from observations in a plantation of Corsican Pine. Agric Meteorol 9, 367384

SAS Institute Inc (1987) SAS/STAT guide for personal computers version 6 edition. SAS Institute Inc Cary, NC, USA, $1030 \mathrm{p}$

Schulze RE, Scott-Shaw CR, Nanni UW (1978) Interception by Pinus patula in relation to rainfall parameters. J Hydro/ 36, 393-396

Stigter K (1987) Puiser dans le savoir traditionnel. Cérès 117, 29-32

Zinke PJ (1967) Forest interception studies in the United States. In: Forest Hydrology Proceedings of a National Science Fondation Advanced Science Seminar, Pennsylvania State University, Aug 29-Sept 101965 (WE Sopper, HW Lull, eds). Pergamon Press, Oxford, 137-161 